\title{
Changes in Cardiac Repolarization Following Short Periods of Ventricular Pacing
}

\author{
RAJIVA GOYAL, M.D., ZAFFER A. SYED, B.E., \\ PARTHA S. MUKHOPADHYAY, B.SC., JOSEPH SOUZA, M.D., \\ ADAM ZIVIN, M.D., BRADLEY P. KNIGHT, M.D., K. CHING MAN, D.O., \\ S. ADAM STRICKBERGER, M.D., and FRED MORADY, M.D. \\ From the Division of Cardiology, Department of Internal Medicine, \\ The University of Michigan Medical Center, Ann Arbor, Michigan
}

Cardiac Memory. Introduction: "Cardiac memory" (primary T wave change) is thought to occur after 15 minutes to several hours of right ventricular (RV) pacing. The two components of the temporal change in repolarization are memory and accumulation. The purpose of this study was to examine quantitatively the effect of short periods of ventricular pacing on the human cardiac action potential, using monophasic action potential (MAP) recordings.

Methods and Results: Thirty-one patients (ages $43 \pm 14$ years) with structurally normal hearts undergoing a clinically indicated electrophysiologic procedure were enrolled. Catheters were placed in the right atrium (RA) and RV, and a MAP catheter was positioned at the RV septum. $\mathrm{APD}_{9_{0}}$ was calculated from digitized MAP recordings. MAP morphology comparisons were performed using the root mean square (RMS) of the difference between complexes. All pacing was at 500-msec cycle length. There were four pacing protocols: (1) RA pacing was performed for approximately 15 minutes to evaluate temporal stability of the MAP recordings (5 pts); (2) to evaluate the memory phenomenon, four successive 1-minute episodes of RV pacing were interspersed with 2 minutes of RA pacing ( 5 pts); (3) the accumulation phenomenon was evaluated by assessing the effects of $1,5,10$, and 15 minutes of $\mathrm{RV}$ pacing on the MAP during RA pacing (16 pts); and (4) 20 minutes of RV pacing was followed by 10 minutes of RA pacing to correlate visually apparent $T$ wave changes with changes in MAP recordings (5 pts). In the control patients, no changes in $\mathrm{APD}_{90}$ or RMS analysis were noted during $14.9 \pm 1.4$ minutes of $\mathrm{RA}$ pacing. In the second protocol, RMS of the difference between the baseline MAP complexes and the signal average of the first $\mathbf{5 0}$ beats following each of four 1-minute $\mathrm{RV}$ pacing trains demonstrated progressively greater differences in morphology after successive episodes of RV pacing. In protocol 3, RMS analysis identified a progressively greater difference between the baseline MAP recording and the average of the first 50 beats after $1,5,10$, and 15 minutes of $\mathrm{RV}$ pacing. In protocol 4, visually apparent changes in $T$ waves occurred in parallel with the RMS of the difference between the baseline MAP recordings and the average of the first 50 beats after 20 minutes of RV pacing. Similar changes also were demonstrated by $\mathbf{A P D}_{90}$ analysis.

Conclusion: This study is the first to demonstrate that episodes of abnormal ventricular activation as short as $\mathbf{1}$ minute in duration may exert lingering effects on the repolarization process once normal ventricular activation resumes. ( $J$ Cardiovasc Electrophysiol, Vol. 9, pp. 269-280, March 1998)

ventricular repolarization, ventricular pacing, cardiac memory, monophasic action potential, autonomic blockade

Address for correspondence: Rajiva Goyal, M.D., Division of Cardiology, University of Michigan Hospital, 1500 East Medical Center Drive, B1-F245, Ann Arbor, MI 48109-0022. Fax: 313936-7026.

Manuscript received 12 December 1997; Accepted for publication 26 January 1998.

\section{Introduction}

Rosenbaum et al. ${ }^{1}$ first coined the term "cardiac memory" to describe primary T wave changes following 15 minutes to several hours of right ventricular (RV) pacing. For this temporal change in 
repolarization to be present, two components are necessary, namely memory and accumulation..$^{2-7} \mathrm{~A}$ true memory component is present when repeated episodes of an altered activation sequence exert a cumulative effect on repolarization during normal activation. Accumulation is the occurrence of progressively greater repolarization changes during normal activation following progressively longer durations of an altered activation sequence. Although it is known that ventricular pacing $>15$ minutes in duration may produce changes in ventricular repolarization, no prior studies have examined quantitatively the magnitude of change or the minimum pacing duration required to produce this type of change. ${ }^{1-7}$ Therefore, the purpose of this study was to examine quantitatively the effect of short periods of ventricular pacing on ventricular repolarization in humans.

\section{Methods}

\section{Study Design}

Traditionally, the memory phenomenon has been evaluated by visually apparent $T$ wave changes on the surface ECG. ${ }^{1}$ However, some prior studies have used transmembrane action potential recordings to investigate cardiac memory. ${ }^{6,8}$ In a finite element computer model, changes in the action potential were compared with a standard template to derive the QRS complex. ${ }^{9}$ The differences between two action potentials has been proposed as a method for deriving the surface QRS complex. ${ }^{10}$ Since the $\mathrm{T}$ wave represents a summation of repolarization of all of the ventricular myocytes, it has been assumed that the action potential may be more sensitive to a change in repolarization. In the present study, monophasic action potential (MAP) recordings were used to look for changes in repolarization. ${ }^{11-17}$ The MAP recordings for each patient at baseline were used as the patient-specific template for the subsequent comparisons. Several studies have confirmed that MAP recordings reflect the transmembrane action potential morphology as measured by cell impalement techniques..$^{12,14}$

Because the action potential duration (APD) varies at different sites in the ventricle, ${ }^{11}$ the MAP catheter was positioned at the RV septum in every patient. The cardiac action potential is affected by changes in rate and by changes in autonomic tone. ${ }^{17,18}$ Therefore, the first three pacing protocols were performed at a constant cycle length of 500 $\mathrm{msec}$, and during pharmacologic blockade of $\beta$ and muscarinic receptors. ${ }^{19}$
The study was designed to evaluate the effect of ventricular pacing on the MAP recorded during atrial pacing. The acquisition and analysis of MAP recordings was performed only during right atrial (RA) pacing, and each patient was his/her control for the study protocol. In each patient, the control recordings were made after MAP catheter stability had been visually validated for 5 minutes during RA pacing. Catheter stability was confirmed fluoroscopically and by visual evaluation of the MAP morphology. All recordings were at least 10 $\mathrm{mV}$ in amplitude. ${ }^{16,20}$

The patients enrolled in the study underwent 1 of 4 protocols (Fig. 1). The first protocol was to evaluate the temporal stability of MAP recordings during RA pacing for 15 minutes. In the second protocol, to evaluate the memory phenomenon, baseline MAPs were recorded for 1 minute during RA pacing. RV pacing then was performed for 1 minute, followed by MAP acquisition during RA pacing for 2 minutes. This pacing sequence was repeated for a total of four RV pacing sequences. MAPs obtained during RA pacing after each 1minute episode of RV pacing were compared to baseline MAPs. The third protocol was performed to evaluate the accumulation phenomenon. After acquisition of baseline MAP recordings for 1 minute during RA pacing, patients underwent 1 of 2 pacing options. One group of patients underwent RV pacing for 1,5 , and 10 minutes interspersed with periods of RA pacing. MAP recordings were acquired only during RA pacing. The duration of RA pacing was varied to allow the MAP duration to return to the baseline value. In the other group of patients, RV pacing for 15 minutes was followed by recording of MAPs during RA pacing for 5 minutes. The pacing protocol used to evaluate accumulation was divided between two groups of patients to avoid a lengthy study protocol in individual patients.

The term "cardiac memory" traditionally has been used to describe $\mathrm{T}$ wave changes following RV pacing. ${ }^{1}$ Therefore, a fourth pacing protocol was performed to correlate $T$ wave changes with changes in the MAP recordings, thereby confirming that the MAP recordings were a valid indicator of altered repolarization as reflected in the T wave. In contrast to the other pacing protocols, RV pacing was performed for 20 minutes in an attempt to bring out visually apparent $\mathrm{T}$ wave changes. In the previous three protocols, RA pacing at a cycle length of $500 \mathrm{msec}$ sometimes resulted in the pacing artifact distorting the $\mathrm{T}$ wave morphology. Therefore, in this portion of the study, to prevent 


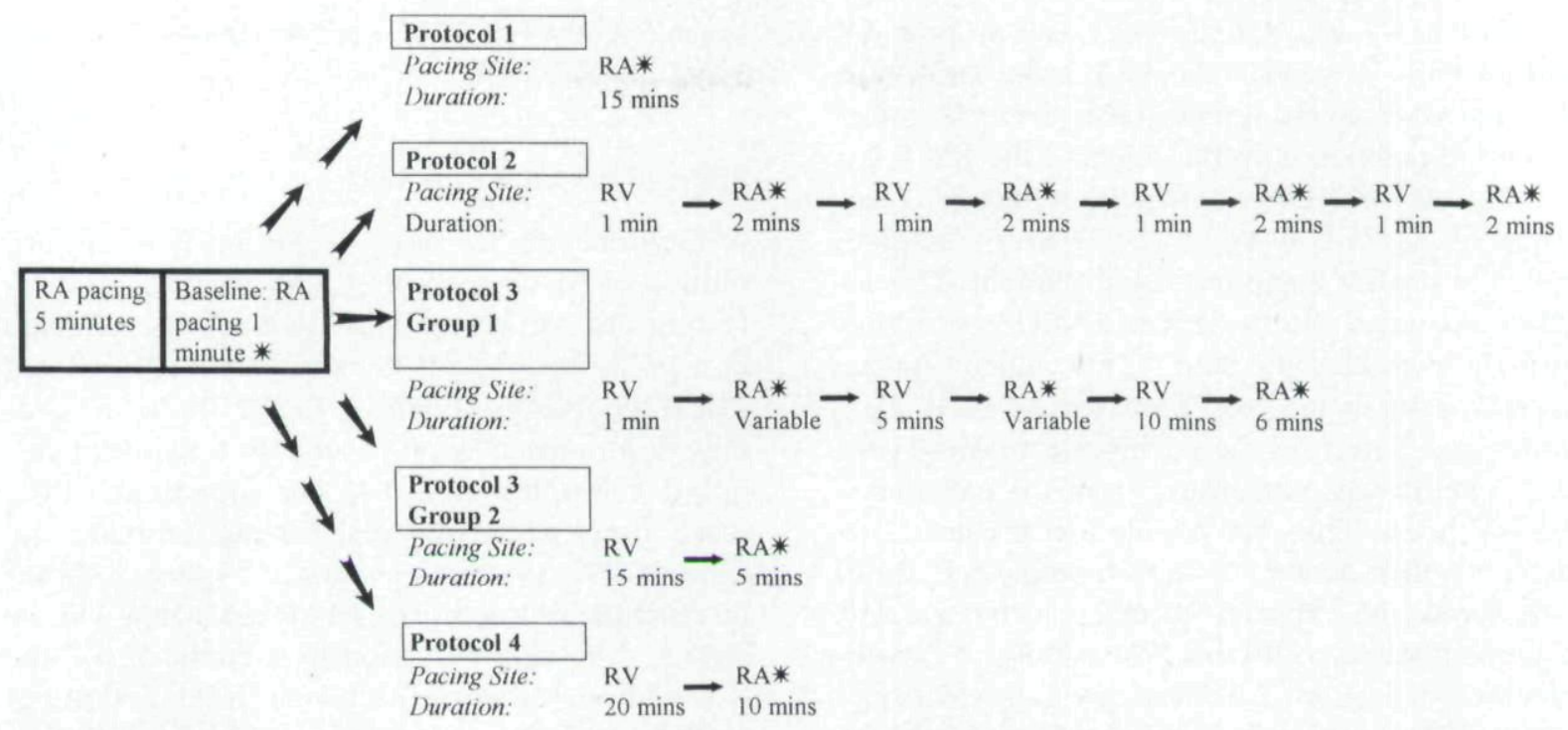

Figure 1. Outline of the study protocols. Protocol 1 was designed to evaluate the temporal stability of the monophasic action potential (MAP) recordings; protocol 2 was designed to evaluate the memory phenomena; protocol 3 was designed to evaluate accumulation; and protocol 4 was designed to correlate $T$ wave changes with the changes noted on the MAP recordings. Pharmacologic autonomic blockade was given prior to protocols 1, 2, and 3, and the pacing cycle length was always 500 msec during the protocols. During protocol 4, autonomic blockade was not present, and the pacing cycle length was 700 msec. There were no pauses when switching from one pacing site to another. The asterisks (*) designate the steps of the protocol during which MAP recordings were acquired. $R A=$ right atrium; $R V=$ right ventricle.

distortion of $\mathrm{T}$ waves, RA and RV pacing were performed at a cycle length of $700 \mathrm{msec}$. Autonomic blockade was not used during this portion of the study, to avoid spontaneous sinus cycle lengths $<700 \mathrm{msec}$.

\section{Patient Characteristics}

The study group consisted of 31 patients who underwent an electrophysiologic procedure for evaluation and treatment of paroxysmal supraventricular tachycardia at the University of Michigan Medical Center. Five patients were enrolled in the control protocol, 5 patients in the memory evaluation protocol, 16 patients in the protocol to evaluate accumulation (10 patients in group 1 and 6 patients in group 2), and 5 patients in the protocol to evaluate the relationship between $\mathrm{T}$ wave change and the change in MAP recordings. Exclusion criteria consisted of atrial fibrillation, a sinus cycle length $<600 \mathrm{msec}$ (for protocols 1,2 , and 3 ), a sinus cycle length $<$ $700 \mathrm{msec}$ (for protocol 4), structural heart disease, the presence of a bundle branch block, the recent occurrence of ventricular preexcitation, or treatment with an antiarrhythmic medication. There were 9 men and 22 women (mean age $43 \pm 14$ years $[ \pm \mathrm{SD}])$.

\section{Electrophysiologic Testing}

After informed consent was obtained, the electrophysiologic procedures were performed with the patients in the fasting state. Quadripolar electrode catheters were positioned in the heart as clinically indicated. The 12-lead ECG and intracardiac electrograms were displayed on an oscilloscope and recorded on paper using a Mingograph 7 recorder (Siemens-Elema, Solna, Sweden). Bipolar pacing was performed using the distal pair of electrodes of the quadripolar catheter, with an interelectrode distance of $2 \mathrm{~mm}$. Pacing was performed with a programmable stimulator (Bloom Associates, Reading, PA, USA) using a stimulus strength three times diastolic threshold and stimulus duration of $2 \mathrm{msec}$.

\section{Study Protocol}

The study protocol was approved by the $\mathrm{Hu}$ man Research Committee and was performed upon completion of the radiofrequency ablation component of the electrophysiologic procedure, during the 30-minute waiting period to confirm a successful outcome. Autonomic blockade was achieved by infusion of propranolol $0.2 \mathrm{mg} / \mathrm{kg}$ and atropine $0.04 \mathrm{mg} / \mathrm{kg}$ during a period of approximately 5 minutes ${ }^{19}$ (protocols 1, 2, and 3). The mean patient 
weight was $81 \pm 13 \mathrm{~kg}$, and the mean propranolol and atropine dosages were $16.1 \pm 3.2$ and $3.4 \pm$ $0.7 \mathrm{mg}$, respectively. Quadripolar electrode catheters were positioned in the RA and the RV apex. A 7-French MAP catheter (model 1675P, EP Technologies, Mountain View, CA, USA) was positioned at the RV septum in every patient. This location provided the most stable MAP recordings over the period of the study. This catheter has an interelectrode distance of $4 \mathrm{~mm}$ for the MAP electrodes and $2 \mathrm{~mm}$ for the pacing electrodes. However, pacing was performed from the catheter at the RV apex. This site resulted in greater QRS aberration than during RV septum pacing and, therefore, would be expected to result in the greatest $\mathrm{T}$ wave change following RV pacing. ${ }^{1,4} \mathrm{~A}$ ventricular electrogram from the "pacing" electrodes of the MAP catheter was recorded in addition to the MAP recording, and this was used as a trigger during later analyses. The endocardial electrograms and surface leads were recorded on paper at $25 \mathrm{~mm} / \mathrm{sec}$ using the Mingograph 7 recorder. The MAP channel was unfiltered, and a bandwidth of 0.05 to $1000 \mathrm{~Hz}$ was used. Output from the recorder was connected to an FM magnetic tape recorder (Teac $130 \mathrm{TE}$, Tokyo Japan) that had a total sampling frequency of $12 \mathrm{kHz}$. Analog-todigital conversion was performed from the digital analog tape at a sampling frequency of $1 \mathrm{kHz}$ per channel, and the data were stored in separate files for later splicing.

\section{APD Evaluation}

On-line measurements of APD were made using a program written for the purpose in the $\mathrm{Vi}$ sual Engineering Environment (Hewlett-Packard, Loveland, CO, USA). APD ${ }_{90}$ was defined as the time interval from the rapid upstroke (phase zero) to the time for the action potential amplitude to decrease to $10 \%$ of the peak plateau amplitude. In a pilot study, 200 MAP recordings were reviewed to validate the accuracy of the computerized measurements. The on-line measurements were used to determine when the $\mathrm{APD}_{90}$ had returned to baseline after RV pacing sequences in the component of the study protocol used to evaluate accumulation. When the $\mathrm{APD}_{90}$ returned to baseline, the next sequence of RV pacing was initiated.

Off-line analysis also was performed on the digitized MAP recording using a program written in Matlab (Mathworks, Natick, MA, USA). Either beat-to-beat analysis or signal averaging of a se- lectable number of MAP complexes was performed using this program.

\section{Effect of Amplitude on APD}

Previous studies have suggested that the amplitude of MAP recording may vary without affecting the $\mathrm{APD}_{90}{ }^{16,20}$ This finding was validated in a preliminary study by analyzing 10 digitized MAP recordings. Each of the 10 MAP recordings was reduced in amplitude by a factor of 0.9 , $0.8,0.7,0.6,0.5$, and 0.4 . The measured $\mathrm{APD}_{90}$ were found to be identical for the template and each of the six smaller signals. Figure 2 shows an example of a template MAP recording and the signals following reduction by a factor of 0.7 and 0.5 . Although there is a change in the action potential amplitude, there is no change in $\mathrm{APD}_{90}$.

\section{Morphology Comparisons of MAP Recordings}

To quantitate the differences between two MAP recordings, $230 \mathrm{msec}$ of digitized data was analyzed using a root mean square (RMS) of the difference. Previous work has suggested that the initial deflection of the MAP recordings is subject to variability. ${ }^{14-16}$ In the morphology comparison, therefore, the first $15 \mathrm{msec}$ of the MAP recordings were not included in the RMS of the difference analysis. This technique previously was found to be useful in identifying differences in QRS morphology.21 Using the trigger channel, $50 \mathrm{MAP}$ recordings were aligned and then averaged to increase the signalto-noise ratio. Although variability in the amplitude of MAP recordings does not affect the APD ${ }_{90}$, a morphology comparison using RMS of the difference is affected by changes in amplitude (Fig. 2). To overcome this problem, the amplitudes of the signal-averaged MAP recordings were normalized prior to RMS evaluation. Based on visual inspection of approximately 300 digitized MAP recordings, RMS of the difference values $>4 \mathrm{mV}$ were found to be indicative of visually apparent changes in morphology of the MAP (Fig. 3).

\section{Analysis of the Change in MAP Recordings}

For each patient in the control protocol, the mean of the first minute of the MAP recordings was compared with the $\mathrm{APD}_{90}$ of the subsequent groups of 20 complexes. Comparisons using the RMS of the difference between the first 50 MAP recordings and a similar segment every 1 minute thereafter also were performed. 


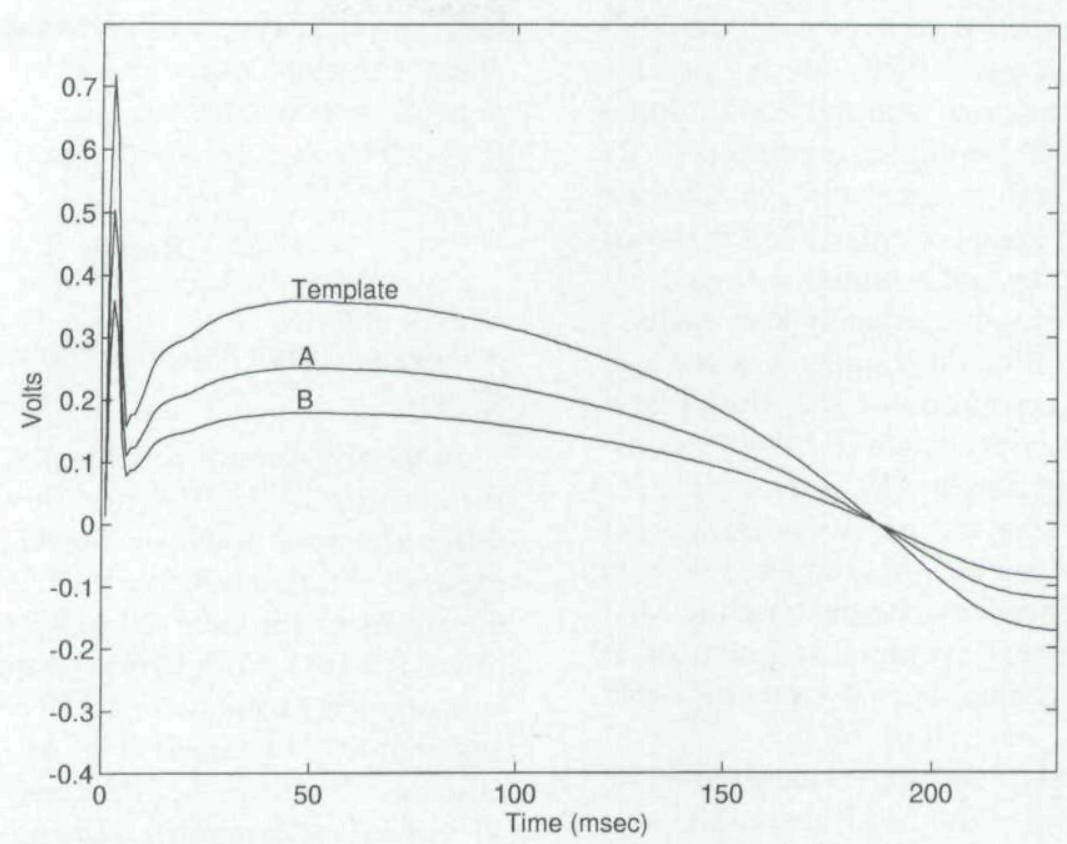

Figure 2. Effect of amplitude on $A P D_{90}$ and root mean square (RMS) of the difference between two monophasic action potential (MAP) complexes. $A P D_{90}$ was defined as the time interval for the action potential amplitude to decrease to $10 \%$ of the peak plateau amplitude. The template MAP (a representative recording from the database) has been reduced in amplitude by a factor of $0.7(A)$ and $0.5(B)$. The $A P D_{90}$ for the three signals is $215 \mathrm{msec}$. Therefore, the change in MAP amplitude does not affect $A P D_{90}$ measurement. The RMS of the difference between the template and MAP recording $A$ is $8 m V$, and the RMS of the difference between the template and MAP recording $B$ is $13 \mathrm{mV}$. As can be seen, the RMS analysis is affected by the amplitude of the signal and, therefore, normalization of amplitude is required before performing this analysis.

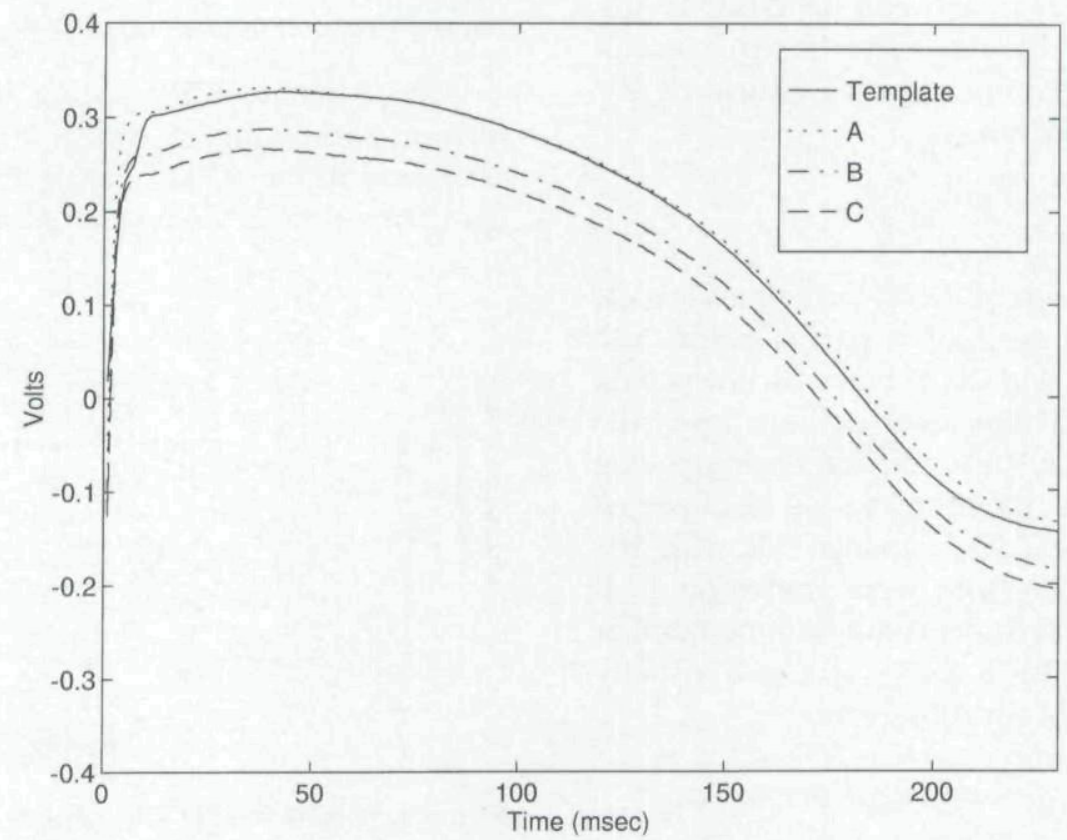

Figure 3. To illustrate the advantage of root mean square (RMS) analysis, a template monophasic action potential (MAP) recording is compared with MAP recordings $A, B$, and $C$. The RMS of the difference values are 1,4, and $6 \mathrm{mV}$, respectively. $A P D_{90}$ for each of the MAP recordings is $206 \mathrm{msec}$. An RMS of the difference $>4 \mathrm{mV}$ was indicative of visually apparent changes in morphology of the MAP recording. 
In the pacing protocol used to evaluate the memory phenomena, the mean $\mathrm{APD}_{90}$ during baseline RA pacing was compared with the $\mathrm{APD}_{90}$ during RA pacing after the 1-minute sequences of RV pacing. RMS of the difference was evaluated between the baseline signal-averaged MAP recordings during RA pacing and a similar averaged segment during RA pacing immediately after each RV pacing sequence. During the 2 minutes of RA pacing, the temporal resolution of the morphology change in MAP was evaluated. RMS of the difference between the baseline MAP recording and a signal-averaged segment of 10 complexes immediately after RV pacing was compared with the RMS of the difference between the baseline MAP recording and a signal-averaged segment of 10 complexes just preceding the subsequent RV pacing sequence.

In pacing protocols 3 and 4 , the change in $\mathrm{APD}_{90}$ was evaluated between the MAP recordings during RA pacing before and after RV pacing. The $\mathrm{APD}_{90}$ of every MAP complex 1 minute prior to RV pacing was evaluated, and a mean baseline $\mathrm{APD}_{90}$ for the 120 complexes was calculated. This value was compared with the $\mathrm{APD}_{90}$ during $\mathrm{RA}$ pacing following the RV pacing sequences. Comparisons of the $\mathrm{APD}_{90}$ following RV pacing were performed using means of consecutive 20-beat groups of MAP complexes. A comparison of the RMS of the difference between the baseline signal-averaged MAP recordings and the MAP recordings during RA pacing upon cessation of RV pacing also was performed.

\section{Visual Comparisons}

Visual comparisons of the $\mathrm{T}$ wave in leads aVF, $\mathrm{V}_{1}, \mathrm{~V}_{4}, \mathrm{~V}_{5}$ and $\mathrm{V}_{6}$ during RA pacing before and after $1,5,10,15$, and 20 minutes of RV pacing were performed. These leads demonstrated the greatest degree of QRS aberration during pacing at the RV apex and, therefore, would be expected to have the greatest $\mathrm{T}$ wave change following RV pacing. ${ }^{1,4}$ The comparisons were graded on a visual score from 0 to 5 , depending on the number of leads in which the $\mathrm{T}$ waves appeared visually indistinguishable by two observers.

\section{Statistical Analysis}

Continuous variables are expressed as mean \pm SD. ANOVA with a multivariate repeated measures design was used when comparing the changes in MAP recordings between more than two groups.
Regression analysis was performed to evaluate the effect of duration of RV pacing on the MAP recordings (Statistica, StatSoft Inc., Tulsa, OK, USA). $\mathrm{P}<0.05$ was considered significant.

\section{Results}

\section{Stability of Action Potential Recordings: Control Protocol}

In the five control patients, RA pacing was performed for $14.9 \pm 1.4$ minutes. No significant difference was noted in the $\mathrm{APD}_{90}$ between the mean of the first 120 beats $(222 \pm 26 \mathrm{msec})$ and the mean of the next 20 MAP beats $(223 \pm 27$ msec; $\mathrm{P}=0.4$ ). Comparison of the subsequent 20beat segments with the first 120 beats did not show any significant changes (Fig. 4). There was a significant decrease in the MAP amplitude over time: $41 \pm 8 \mathrm{mV}$ at 1 minute, $32 \pm 7 \mathrm{mV}$ at 5 minutes, and $18 \pm 4 \mathrm{mV}$ at 10 minutes $(\mathrm{P}<0.02)$. However, using the RMS of the difference analysis between the signal average of the first 50 MAP complexes and similar normalized segments at 1, 5, 10 , and 15 minutes of RA pacing, there were no significant changes in MAP morphology (3.2 \pm $0.7,3.4 \pm 0.2,3.5 \pm 0.2$, and $3.2 \pm 0.2 \mathrm{mV}$, respectively; $\mathrm{P}=0.4$ ).

\section{Pacing Protocol to Demonstrate Memory}

After 1 minute of RV pacing, there was no significant shortening of $\mathrm{APD}_{90}$ during RA pacing compared to the $\mathrm{APD}_{90}$ during baseline RA pacing ( $223 \pm 4$ vs $226 \pm 5 \mathrm{msec} ; \mathrm{P}=0.1)$. Compared

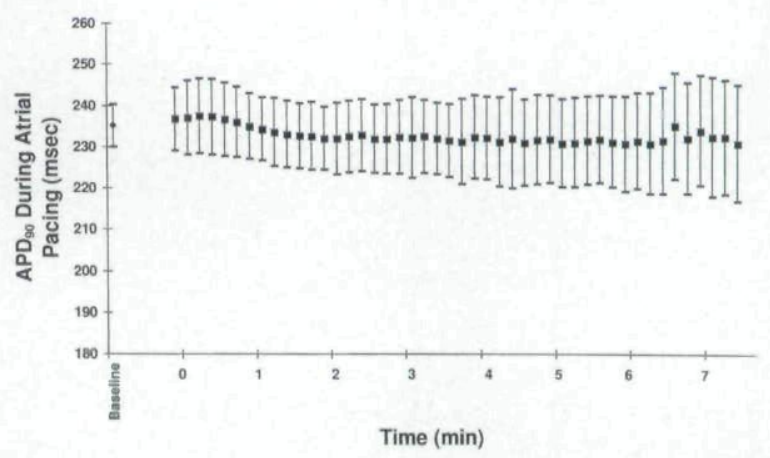

Figure 4. Stability of $A P D_{90}$ during right atrial pacing in five patients. The first data point represents the mean of the first 1 minute of monophasic action potential recording. This is compared with the subsequent 20-beat groups. No significant differences in $A P D_{90}$ occurred during atrial pacing for up to approximately 14 minutes. $* P<0.05$; bars $=1 S D$. 


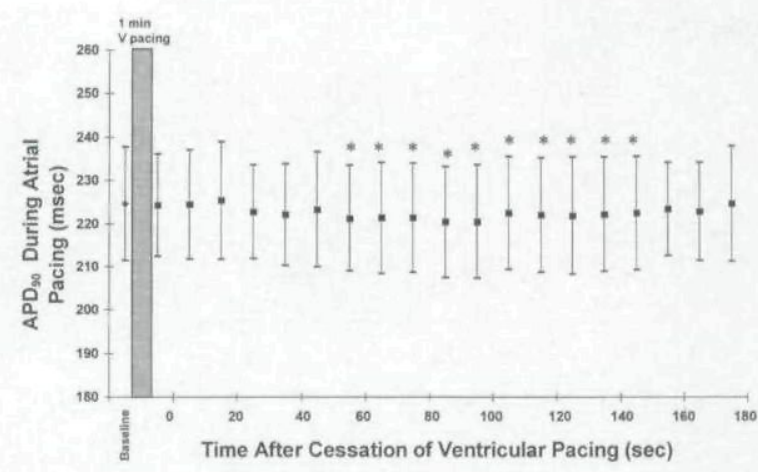

A

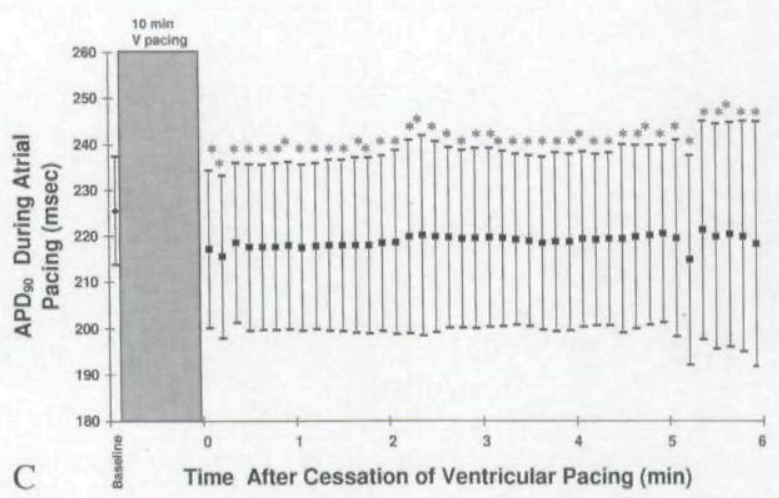

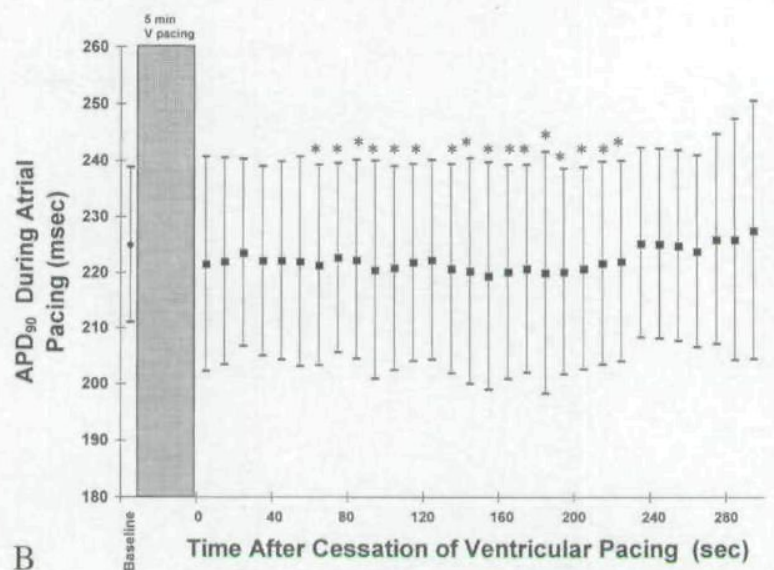

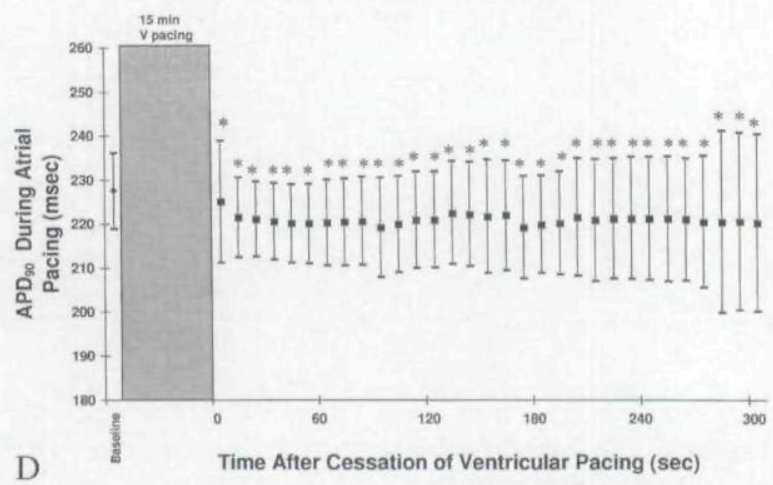

Figure 5. $A P D_{90}$ during right atrial pacing before and after $1(A), 5(B), 10(C)$, and $15(D)$ minutes of $R V$ pacing. The baseline value represents the mean $A P D_{90}$ of 120 monophasic action potential complexes. This baseline is compared with the subsequent 20-beat groups for an approximate duration of 4 to 7 minutes after resumption of atrial pacing. $* P<0.05$. The width of the bar representing ventricular pacing is not to scale.

to baseline, there was a progressive shortening of the $\mathrm{APD}_{90}$ after the second, third, and fourth 1minute episodes of RV pacing $(216 \pm 6,211 \pm 4$, and $207 \pm 5 \mathrm{msec}$, respectively; $\mathrm{P}<0.05$ ).

The RMS of the difference between the baseline MAP recording and the signal average of the first 50 beats following each RV pacing train demonstrated a difference in morphology after each of the four RV pacing trains $(6.3 \pm 0.3,7.7 \pm 0.3$, $9 \pm 0.5$, and $9.4 \pm 0.6 \mathrm{mV}$, respectively). Using regression analysis, a progressively greater change in the RMS of the difference was noted after each of the four RV pacing trains $(r=0.96, P=0.02)$.

The RMS of the difference between baseline and the signal average of the 10 MAP recordings just prior to the second, third, and fourth periods of RV pacing did not reveal a difference in morphology.

\section{Ventricular Repolarization Accumulation}

A significant shortening of the $\mathrm{APD}_{90}$ during RA pacing was noted approximately 60 seconds after the cessation of 1 and 5 minutes of RV pacing (Figs. 5A and 5B). By approximately $150 \mathrm{sec}-$ onds of RA pacing, the $\mathrm{APD}_{90}$ was no longer shorter than baseline. Shortening of $\mathrm{APD}_{90}$ during RA pacing also was noted after 10 and 15 minutes of RV pacing compared to baseline RA pacing (Figs. 5C and 5D). Examples of a baseline MAP during RA pacing before RV pacing and MAP complexes during RA pacing after 1,5 , and 10 minutes of RV pacing are shown in Figure 6.

RMS of the difference analysis noted a change in the MAP morphology after 1 minute of RV pacing $(4 \pm 0.2 \mathrm{mV})$. This difference in morphology was greater after longer durations of RV pacing (Fig. 7).

\section{Visual Analysis}

Compared to the $\mathrm{T}$ waves during baseline RA pacing, no visually apparent changes in $T$ wave morphology during RA pacing were noted after 1 , 5,10 or 15 minutes of RV pacing (visual scores $5 \pm 0,4.9 \pm 0.3,5 \pm 0$ and $4.9 \pm 0.3$, respectively; 
A
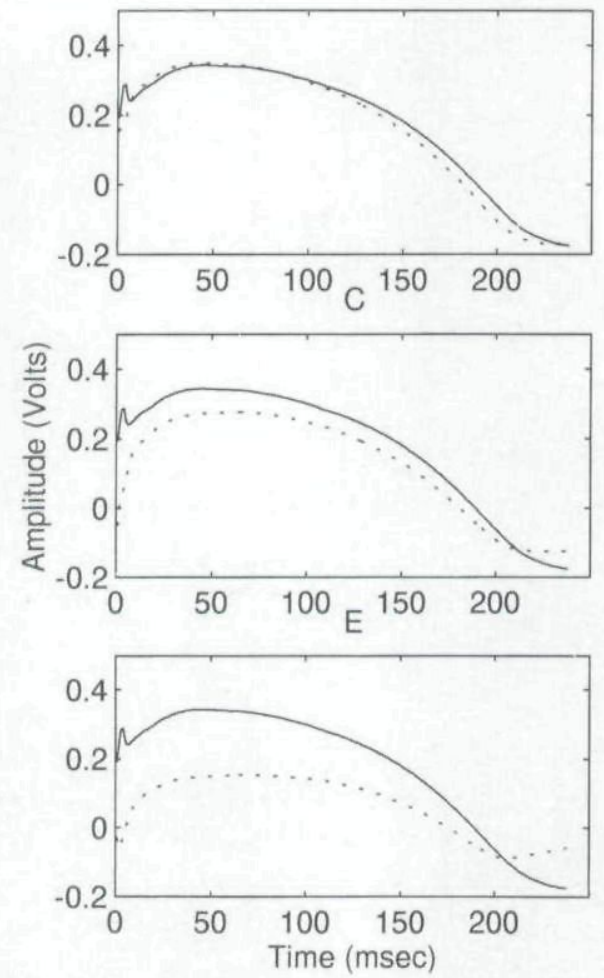

B
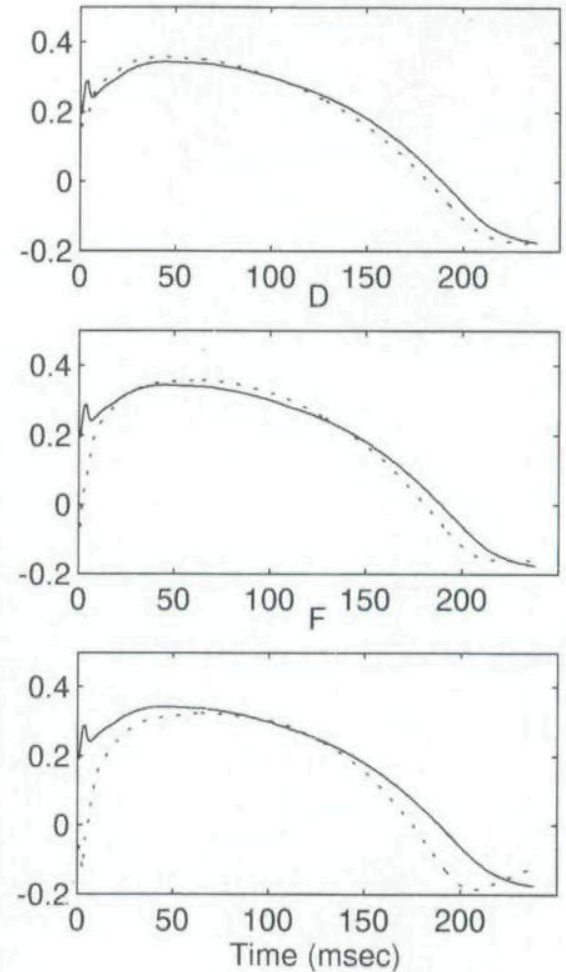

Figure 6. Examples of a monophasic action potential (MAP) recording obtained during baseline (bold line) right (RA) atrial pacing. Plots $A, C$, and $E$ are the MAP recordings (dashed line) without normalization during RA pacing after 1,5 , and 10 minutes of right ventricular $(R V)$ pacing, respectively. All figures are obtained using a signal average of 50 MAP recordings. Plots $B, D$, and $F$ are the normalized MAP recordings (dashed line) of plots $A, C$, and $E$, respectively. The APD $D_{90}$ for baseline, and after 1, 5, and 10 minutes of RV pacing are 227, 215, 209, and $195 \mathrm{msec}$, respectively. The values with and without normalization are identical. The root mean square (RMS) of the difference between control and the MAP recordings without normalization after 1, 5, and 10 minutes of $R V$ pacing are 3, 6, and $17 \mathrm{mV}$. The RMS of the difference (normalized) between control and the MAP recordings after 1,5 , and 10 minutes of $R V$ pacing are 3,4 , and $8 \mathrm{mV}$. There is a decrease in MAP amplitude over time as noted in plots $A, C$, and $E$ (dashed line).

$P=0.4)$. No significant interobserver variability was noted (visual scores $4.8 \pm 0.4$ vs $4.9 \pm 0.1$ for the two observers; $\mathrm{P}=0.5$ ).

\section{Correlation Between $T$ Waves and MAP Recordings}

Compared to baseline there was a significant change in $\mathrm{T}$ wave morphology after 20 minutes of RV pacing (visual scores $1 \pm 0.8$ immediately after, $1.6 \pm 1.3$ after $1 \mathrm{~min}$, and $3.4 \pm 0.5$ after $5 \mathrm{~min}$ were all significantly different from baseline; $\mathrm{P}<$ 0.05 [Fig. 8]). Eight minutes after RV pacing, visually apparent changes in the $\mathrm{T}$ waves were no longer present (visual score $4.6 \pm 0.5 ; \mathrm{P}=0.4$ ).

In parallel with the $\mathrm{T}$ wave changes, there was a significant decrease in $\mathrm{APD}_{90}$ that persisted for the 10 minutes of RA pacing following $20 \mathrm{~min}$ utes of RV pacing ( $255 \pm 13 \mathrm{msec}$ baseline vs 246 $\pm 16 \mathrm{msec}$ immediately after, $244 \pm 17 \mathrm{msec}$ af- ter $5 \mathrm{~min}$, and $245 \pm 18 \mathrm{msec}$ after $10 \mathrm{~min} ; \mathrm{P}<$ 0.02). The RMS of the difference between the baseline MAP recordings and the signal average of 50 beats immediately, and 1,5 , and 10 minutes after RV pacing were all significantly different $(5 \pm$ $0.2,5 \pm 0.1,5 \pm 0.1$, and $4 \pm 0.1 ; \mathrm{P}<0.02$ ).

\section{Discussion}

\section{Main Findings}

Prior studies have used T wave changes to study the memory phenomenon and have found that ventricular pacing for $>15$ minutes is needed to demonstrate memory. ${ }^{1}$ In contrast, in the present study, using $\mathrm{APD}_{90}, 1$ minute of RV pacing was sufficient to create a transient change in repolarization during subsequent RA pacing, while no visually apparent changes were seen in the T wave. RMS of 


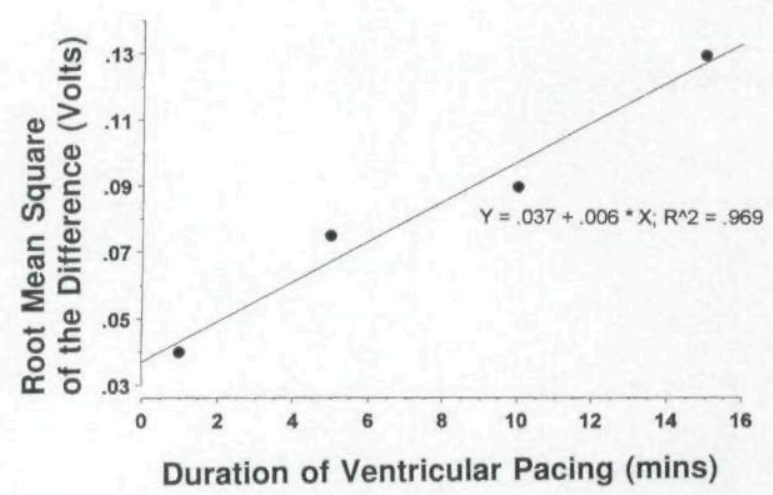

Figure 7. Regression plot of duration of ventricular pacing versus the difference in morphology between the signal-averaged monophasic action potential recordings at baseline and the recordings during right atrial pacing after right ventricular pacing for $1,5,10$, and 15 minutes $(r=0.97, P$ $<0.01)$. Root mean square of the difference $>4 \mathrm{mV}$ identifies a different morphology.

the difference analysis, which also was more sensitive than the $\mathrm{T}$ wave, demonstrated a change in repolarization during atrial pacing immediately after 1 minute of RV pacing. Using the $\mathrm{APD}_{90}$ of the MAP and the RMS of the difference analysis, the criteria of memory and accumulation were satisfied. In other words, pacing the ventricle for repeated 1-minute sequences interspersed with 2 minutes of atrial pacing resulted in progressively greater change in ventricular repolarization during the atrial pacing (memory), and longer durations of ventricular pacing resulted in greater changes in ventricular repolarization during atrial pacing (accumulation). Autonomic effects cannot explain these changes, since the first three pacing protocols were performed under blockade of $\beta$ and muscarinic receptors. Also of note is that these changes were not cycle length dependent, since all pacing was at a constant and uninterrupted cycle length of $500 \mathrm{msec}$ during the first three pacing protocols.

\section{Correlation Between $T$ Wave Changes and MAP Recordings}

In this study, visually apparent $\mathrm{T}$ wave changes were noted only after 20 minutes of RV pacing. These visually apparent changes resolved by 8 minutes after RV pacing. The changes in the MAP recordings (APD ${ }_{90}$ and RMS of the difference) occurred in parallel with the $\mathrm{T}$ wave changes, but persisted for a longer period of time. Therefore, the MAP recordings were more sensitive to the change in repolarization once normal ventricular activation resumed, after a period of abnormal ventricular activation.

\section{Cardiac Memory}

The finding that successive episodes of RV pacing of the same duration have a progressively greater effect on repolarization following the restoration of a normal activation sequence is similar to the findings of previous studies. ${ }^{1,6,7}$ However, this study is the first to demonstrate that such changes in repolarization can take place with a duration of ventricular pacing as short as 1 minute. In addition to this phenomenon, the present study documented the phenomenon of accumulation, with progressively greater alterations in the MAP recordings during atrial pacing following progressively longer periods of ventricular pacing between 1 and 15 minutes in duration.

\section{Delayed Onset of $A P D_{90}$ Shortening}

In this study, a shortening of the $\mathrm{APD}_{90}$ following 1 and 5 minutes of RV pacing was observed after approximately 60 seconds of RA pacing. Following 10 and 15 minutes of RV pacing, there was no delay in the shortening of the $\mathrm{APD}_{90}$. A possible explanation for this finding might be that there are two phenomena present, one that results in a decrease and the other in an increase in $\mathrm{APD}_{90}$. The cardiac accumulation phenomena results in a decrease in $\mathrm{APD}_{90}$, and this decrease is of greater magnitude as the duration of ventricular pacing increases. Previous work has suggested that myocardial stretch can result in activation of stretch-activated ion channels that may result in prolongation of $\mathrm{APD}_{90}{ }^{22.23}$ The time course and magnitude of change in $\mathrm{APD}_{90}$ may differ for the two effects following RV pacing. The results of this study suggest that after 1 and 5 minutes of RV pacing, the competing effects on $\mathrm{APD}_{90}$ are balanced and, therefore, there is no significant change in $\mathrm{APD}_{90}$ immediately upon cessation of RV pacing. By 60 seconds after the cessation of pacing, transient prolongation of $\mathrm{APD}_{90}$ due to stretch or some other mechanism has decreased to a sufficient degree to allow shortening of the $\mathrm{APD}_{90}$ to become manifest. This phenomenon has not been described previously, probably because the prior techniques used to study repolarization were not as sensitive as those in the present study.

\section{Analysis of MAPs}

Conventionally, $\mathrm{APD}_{90}$ has been used to quantify MAP recordings. ${ }^{13}$ However, analysis of APD at other degrees of repolarization also could be used instead of $\mathrm{APD}_{90}$. The results of this study 


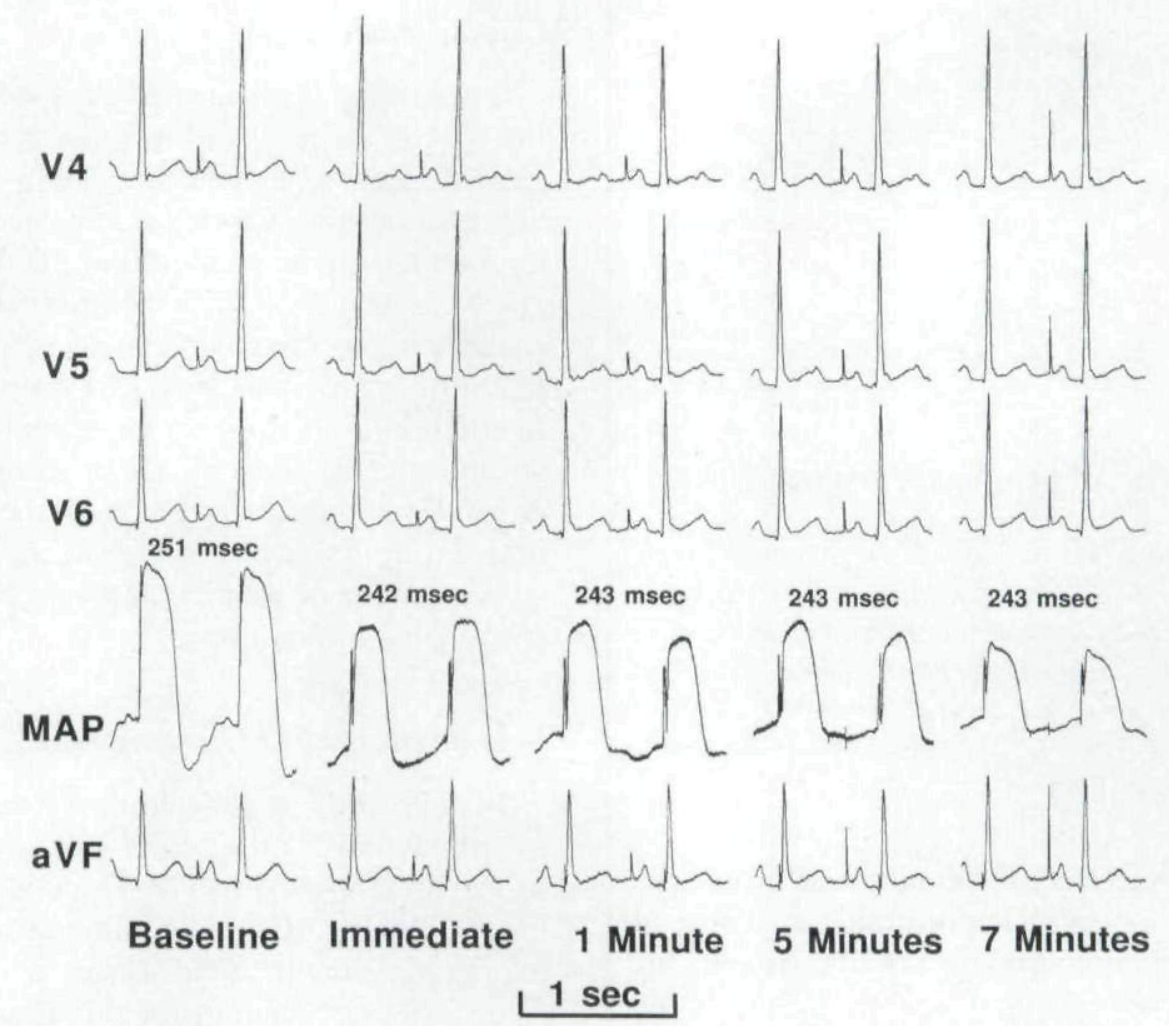

Figure 8. An example of visually apparent changes in the T waves, comparing the baseline ECG with that immediately and 1 , 5 , and 8 minutes after 20 minutes of right ventricular pacing. Shown are surface leads $V_{4}, V_{5}, V_{6}$, aVF, and monophasic action potential (MAP) recording. APD 90 values are shown above the respective MAP recordings. Visual score comparing the baseline to the ECG immediately, and 1, 5, and 7 minutes after are 0,2, 2, and 3 of 4 identical, respectively.

suggest that RMS of the difference between two MAP recordings may be more sensitive than $\mathrm{APD}_{90}$ in detecting changes in the MAP. In this study, RMS of the difference analysis was able to demonstrate a change in MAP recordings after 1 minute of RV pacing, when no difference was detectable by $\mathrm{APD}_{90}$. The RMS analysis at a sample frequency of $1 \mathrm{kHz}$ is able to detect differences at $1-\mathrm{msec}$ intervals and, therefore, may detect a change in MAP when there is no change in the $\mathrm{APD}_{90}$.

\section{Possible Mechanisms}

The change in the MAP recordings demonstrated in this study represent a change in phases 2 and 3 of the cardiac action potential. Geller and Rosen ${ }^{6}$ described changes in APD suggestive of memory in canine epicardial and endocardial slabs. The changes were abolished when 4-aminopyridine (4AP) was used in the tissue bath. 4-AP has been shown to block the transient outward current $\mathrm{I}_{\mathrm{t},}{ }^{24,25}$ They concluded that the $\mathrm{I}_{\mathrm{t}}$ channel, which has a predominant effect on phase 1 of the cardiac action potential and therefore modulates phases 2 and 3, may be involved in the generation of cardiac memory. In the study by Geller and Rosen, ${ }^{6}$ shortening of the APD was noted in recordings from the endocardial tissue. Epicardial tissue slices had action potential prolongation during demonstration of the memory phenomenon. In the present study, we made recordings from the endocardial surface, and this may explain why only shortening of $\mathrm{APD}_{90}$ was noted.

Other investigators have identified a subpopulation of cells deep in the ventricular myocardium that have a predominant $\mathrm{I}_{\mathrm{to}}$ current. ${ }^{26,27}$ The role of these cells in the memory phenomenon remains to be determined. $\mathrm{Katz}^{28}$ has suggested that changes may take place at the cellular level; for example, changes in the channel numbers or types, or a change in the number of gap junctions or their kinetics, might explain the memory phenomenon.

In a previous study, using a mathematical model, a shortening of ventricular repolarization during normal activation was demonstrated following only 20 seconds of an altered activation sequence. ${ }^{9}$ It was noted that reactively coupled cells, rather than 
those coupled purely by resistive dynamics, may be involved in the phenomenon of cardiac memory. The model did not demonstrate any delay in the onset of the repolarization changes, as noted in the current study. The effect of stretch was not modeled in this mathematical system, and this might explain the difference in the findings.

\section{Limitations}

A limitation of this study may be that a mean of 20 to 50 MAP complexes was used for comparisons of the MAP duration and morphology. Therefore, short-lived changes in the MAP may not have been detected. The reason for using a mean of 20 to 50 consecutive complexes was to have more stable values. Another limitation of the study is that the RMS of the difference analysis does not allow determination of whether changes in the MAP are the result of prolongation or shortening of the MAP. Previous studies in animal models have evaluated the phenomenon of cardiac memory by comparing endocardial and epicardial action potentials. ${ }^{5,7}$ In the current study, recordings of epicardial action potentials were not feasible since the MAP recordings were obtained transvenously. Therefore, endocardial MAP recordings before an intervention were used as the control to document any change following interventions. It has been noted previously that the direction of changes in endocardial and epicardial action potentials after periods of abnormal depolarization usually are opposite. ${ }^{7}$ Therefore, the changes in endocardial MAP duration noted in this study are highly likely to reflect concomitant changes in the epicardial MAP.

\section{Conclusion}

The results of this study demonstrate that even short episodes of abnormal ventricular activation may exert lingering effects on the repolarization process once normal ventricular activation resumes, and that these effects are independent of changes in the rate of depolarization. The results obtained with ventricular pacing in this study may be applicable to spontaneous episodes of ventricular tachycardia and may provide a possible explanation for how an episode of ventricular tachycardia may predispose to early recurrences. If additional studies demonstrate a similar phenomenon in the atrium, this also may provide an explanation for how atrial fibrillation shortens atrial refractoriness and predisposes to further episodes of atrial fibrillation. ${ }^{29}$

\section{References}

1. Rosenbaum MB, Blanco HH, Elizari MV, et al: Electrotonic modulation of the $\mathrm{T}$ wave and cardiac memory. Am J Cardiol 1982;50:213-222.

2. Luy G, Bahl OP, Massie E: Intermittent left bundle branch block: A study of the effects of left bundle branch block on ECG patterns of MI and ischemia. Am Heart J 1944;28:332-347.

3. Engel JR, Shah R, DePodesta LA, et al: T wave abnormalities of intermittent left bundle branch block. Ann Intern Med 1978;89:204-206.

4. Kalbfleisch SJ, Sousa J, El-Atassi R, et al: Repolarization abnormalities after ablation of accessory atrioventricular connections with radiofrequency current. J Am Coll Cardiol 1991;18:1761-1766.

5. Autenrieth G, Surawicz B, Kuo CS, et al: Primary T wave abnormalities caused by uniform and regional shortening of ventricular monophasic action potential in dog. Circulation 1975;51:688-676.

6. Geller JC, Rosen MR: Persistent T wave changes after alteration of ventricular activation sequence: New insights into cellular mechanisms of "cardiac memory." Circulation 1993;88:1811-1819.

7. del Balzo U, Rosen MR: $T$ wave changes persisting after ventricular pacing in canine hearts are altered by 4 aminopyridine but not by lidocaine: Implications with respect to phenomenon of cardiac "memory." Circulation 1992;85:1464-1472.

8. Costard-Jackle A, Goetsch B, Antz M et al: Slow and long-lasting modulation of myocardial repolarization produced by ectopic activation in isolated rabbit hearts: Evidence for cardiac "memory." Circulation 1989;80: 1412-1420.

9. Goyal R, Hero A III, Morady F: Simulation of cardiac memory in a computer model utilizing reactive coupling. J Electrocardiol 1995;28:180-183.

10. Wohlfart B: Simulation of ECG from two pairs of action potentials. Clin Physiol 1993;13:453-467.

11. Franz MR, Bargheer K, Rafflenbeul W, et al: Monophasic action potential mapping in human subjects with normal electrocardiograms: Direct evidence for the genesis of the T wave. Circulation 1987;75:379-386.

12. Hoffman BF, Cranefield PF, Lepeschkin E: Comparison of cardiac monophasic action potentials recorded by intracellular and suction electrodes. Am J Physiol 1959;196:1297.

13. Franz MR, Burkhoff D, Spurgeon $\mathrm{H}$, et al: In vitro validation of a new cardiac catheter technique for recording monophasic action potentials. Eur Heart J 1986; 7:34-41.

14. Ino T, Karagueuzian HS, Hong K, et al: Relation of monophasic action potential recorded with contact electrode in underlying transmembrane action potential properties in isolated cardiac tissues: A systematic microelectrode validation study. Cardiovasc Res 1988; 22:255-264.

15. Franz MR: Method and theory of monophasic action potential recording. Prog Cardiovasc Dis 1991;33:347-368. 
16. Franz MR: Long term recording of monophasic action potentials from human endocardium. Am J Cardiol 1983;51:1629-1634.

17. Denes P, Wu D, Dhingra R, et al: The effects of cycle length on cardiac refractory periods in man. Circulation 1974;49:32-41.

18. Morady F, Kadish AH, Toivonen LK, et al: The maximum effect of an increase in rate on human ventricular refractoriness. PACE 1988;11:2223-2234.

19. Jose AD, Taylor RR: Autonomic blockade by propranolol and atropine to study intrinsic myocardial function in man. J Clin Invest 1969;48:2019-2031.

20. Lee RJ, Liem LB, Cohen TJ, et al: Use dependent effect of class I antiarrhythmic drugs on the relationship between action potential duration and refractoriness in the human heart. Circulation 1989;80(Suppl II): 327 .

21. Goyal R, Harvey M, Daoud EG, et al: Effect of coupling interval and pacing cycle length on morphology of paced ventricular complexes: Implications for pace mapping. Circulation 1996;94:2843-2849.

22. Akay M, Craelius W: Mechanoelectrical feedback in cardiac myocytes from stretch-activated ion channels. IEEE Trans Biomed Eng 1993;40:811-816.
23. Craelius W: Stretch-activation of rat cardiac myocytes. Exp Physiol 1993;78:411-423.

24. Nakayama T, Irisawa H: Transient outward current carried by potassium and sodium in quiescent atrioventricular node cells of rabbits. Circ Res 1985;57:65-73.

25. Bowma WC, Savage AO: Pharmacological actions or aminopyridines and related compounds. Rev Pure Appl Pharmacol Sci 1981;2:317-371.

26. Litovsky SH, Antzelevitch C: Rate dependence of action potential duration and refractoriness in canine ventricular endocardium differs from that of epicardium: Role of the transient outward current. J Am Coll Cardiol 1989;14:1053-1066.

27. Sicouri S, Antzelevitch C: A subpopulation of cells with unique electrophysiological properties in the deep subepicardium of the canine ventricle. The M cell. Circ Res 1991;68:1729-1741.

28. Katz MK: T wave "memory": Possible causal relationship to stress-induced changes in cardiac ion channels? J Cardiovasc Electrophysiol 1992;3:150-159.

29. Wijffels MCEF, Kirchhof CJHJ, Dorland R, et al: Atrial fibrillation begets atrial fibrillation: A study in awake chronically instrumented goats. Circulation 1995; 92:1954-1968. 
This document is a scanned copy of a printed document. No warranty is given about the accuracy of the copy. Users should refer to the original published version of the material. 